\title{
Declarações de óbitos de mulheres em idade fértil: busca por óbitos maternos
}

\author{
Death certificates of women in childbearing age: search for maternal deaths
}

Certificados de defunción de mujeres en edad fértil: búsqueda por muertes maternas

\author{
Mariana Marcos Gil', Flavia Azevedo Gomes-Sponholz" \\ ' Universidade de São Paulo, Escola de Enfermagem de Ribeirão Preto, \\ Programa de Pós-Graduação Enfermagem em Saúde Pública (Mestranda). Ribeirão Preto-SP, Brasil. \\ "Universidade de São Paulo, Escola de Enfermagem de Ribeirão Preto, \\ Programa de Pós-Graduação Enfermagem em Saúde Pública. Ribeirão Preto-SP, Brasil.
}

Submissão: 21-03-2012 Aprovação: 21-05-2013

\section{RESUMO}

No Brasil, há deficiência de registros completos na Declaração de Óbito, sendo questionada a confiabilidade, especialmente das causas relacionadas ao ciclo grávido-puerperal. Investigamos, em Declarações de Óbito de mulheres em idade fértil, o preenchimento dos campos que permitem identificar óbitos maternos. Pesquisa documental, conduzida a partir de prontuários hospitalares. Analisamos mortes maternas declaradas, não maternas, inconclusivas e mortes presumíveis. Para análise das causas básicas de morte utilizamos a Lista de Tabulação de Mortalidade da CID - BR-10. Das 301 declarações de óbito analisadas, 60\% apresentaram os campos 43/44 preenchidos, e 40\% apresentaram estes campos em branco e/ou ignorados. Encontramos 58,5\% de mortes não maternas, 2\% de mortes maternas declaradas e 39,5\% mortes inconclusivas. A análise das mortes inconclusivas permitiu-nos classificar 4,3\% como mortes presumíveis. Para sanar as incompletudes dos registros civis, é necessário o empenho de todos os profissionais de saúde para que a fidedignidade das informações seja uma meta atingida.

Descritores: Mortalidade Materna; Saúde da Mulher; Subregistro.

\section{ABSTRACT}

In Brazil, there is a lack of complete records on death certificates, and its reliability is questioned, especially for causes attributed to pregnancy and childbirth. We investigated, based on death certificates of women in reproductive age, any fields for identifying maternal deaths. Documentary research, conducted in hospital records. We analyzed in death certificates, maternal and no maternal deaths, inconclusive deaths and hidden deaths. To analyze the underlying causes of death we used ICD 10th Revision. Of the 301 death certificates reviewed, $60 \%$ had the fields $43 / 44$ completed, and $40 \%$ had these fields blank and/or ignored. We found $58.5 \%$ of no maternal deaths, $2 \%$ of maternal deaths and $39.5 \%$ inconclusive. The analysis of inconclusive deaths allowed us to classify $4.3 \%$ as hidden deaths. To overcome the incompletitudes of civil registries, it is necessary that all health professionals be committed to the reliability of the information, so the priority target could be reached.

Key words: Maternal Mortality; Women's Health; Under Registration.

\section{RESUMEN}

En Brasil, hay falta de registros completos sobre los certificados de defunción, y la fiabilidad, especialmente por causas relacionadas con el embarazo y puerperio he sido cuestionado. Investigamos, con base en los certificados de defunción de mujeres en edad fértil, los campos para la identificación de las muertes maternas. Investigación documental, realizada a partir de registros hospitalarios. Se analizaron los certificados de defunción, muertes maternas y no maternas, no conclusivas y presumibles. Para analizar las causas subyacentes de la muerte utilizamos la CIE 10 Revisión. De los 301 certificados de defunción revisados, 60\% tenían campos 43/44 completos, 40\% tenían estos campos en blanco y/o ignoradas. Se encontró $58,5 \%$ muertes no maternas, $2 \%$ de muertes maternas y $39,5 \%$ no conclusivas. El análisis de las muertes no conclusivas nos ha permitido clasificar 4,3\% de muertes presumibles. Para superar las incompletitudes de los registros civiles, es necesario que todos los profesionales de la salud estén comprometidos con la fiabilidad de la información.

Palabras clave: Mortalidad Materna; Salud de la Mujer; Omisiones de Registro.

\section{AUTOR CORRESPONDENTE Flavia Gomes-Sponholz E-mail: flagomes@eerp.usp.br}




\section{INTRODUÇÃO}

Todos os dias, aproximadamente 1500 mulheres morrem, no mundo todo, por complicações relacionadas ao ciclo gravídico-puerperal; mais de 99\% destas mortes ocorrem nos países em desenvolvimento ${ }^{(1)}$. A mortalidade materna é utilizada como parâmetro para avaliar a qualidade dos serviços de saúde oferecidos à população. Desta forma, quanto menor a razão de mortalidade materna de uma região ou país, melhor a qualidade da atenção à saúde( ${ }^{(2)}$.

Para a notificação internacional de óbitos de mulheres no ciclo grávido-puerperal padronizou-se a utilização da razão de mortalidade materna. A Declaração de Óbito, instrumento utilizado na obtenção de dados sobre mortes maternas, é o documento oficial que atesta a morte de uma pessoa, cumpre as exigências legais de registro dos óbitos e é fonte de dados para estatísticas sanitárias. É especialmente importante no que se refere à vigilância epidemiológica da morte materna, servindo como ponto de partida para as investigações ${ }^{(3)}$.

Desde 1995, o Ministério da Saúde incluiu na Declaração de Óbito dois campos referentes a óbitos de mulheres em idade fértil. O campo de número 43 diz respeito à investigação da ocorrência do óbito durante a gravidez, parto ou aborto e o campo de número 44 refere-se à morte ocorrida durante o puerpério. Estes dois campos se destinam a qualificar as condições e causas que provocaram a morte.

O preenchimento da Declaração de Óbito de mulheres em idade fértil também deve conter informações detalhadas sobre a causa básica da morte com o propósito de possibilitar a identificação das causas diretas ou indiretas de mortes maternas. Desta forma, favorece-se a obtenção de dados confiáveis e comparáveis sobre a morte ${ }^{(4)}$.

Apesar de todas as tentativas para melhorar a qualidade das estatísticas oficiais de mortes maternas, ainda existem problemas no que se refere à Declaração de Óbito. Um deles é a subnotificação das mortes, que pode estar relacionada à incorreções técnicas no preenchimento das Declarações de Óbito ${ }^{(5)}$ e configura-se em um dos pontos críticos para a identificação de óbitos maternos, pois compromete a qualidade da informação e o conhecimento dos determinantes das mortes.

Para as causas maternas a subnotificação é elevada, mesmo que haja indícios de que o óbito esteja relacionado ao ciclo grávido-puerperal. Na maioria dos casos, não se menciona, na Declaração de Óbito, que se tratava de uma gestante ou puérpera ${ }^{(4)}$.

Desta forma, propusemos este estudo, que teve como cenário o Hospital das Clínicas da Faculdade de Medicina de Ribeirão Preto da Universidade de São Paulo, e cujo objetivo é investigar, em Declarações de Óbito de mulheres de 10 a 49 anos, o preenchimento dos campos que permitem a identificação de óbitos maternos e classificar os óbitos quanto à sua natureza.

A definição de morte materna utilizada foi a da Classificação Internacional de Doenças - $10^{\mathrm{a}}$ Revisão, que considera morte materna a morte de uma mulher durante a gestação ou até 42 dias após o término da gestação, independente da duração ou da localização da gravidez, devida a qualquer causa relacionada com ou agravada pela gravidez ou por medidas em relação a ela, porém não devida a causas acidentais ou incidentais ${ }^{(6)}$.
Quanto à natureza do óbito, consideramos como morte materna declarada quando as informações registradas na Declaração de Óbito permitiram classificar o óbito como materno. Isto significa que constava na Declaração de Óbito uma causa específica do capítulo XV - Gravidez, parto e puerpério, da Classificação Internacional de Doenças - $10^{\mathrm{a}}$ revisão, ou havia menção ao estado gravídico-puerperal, independente de ter os campos correspondentes aos óbitos de mulheres preenchidos ${ }^{(5)}$.

Consideramos morte não materna quando as informações registradas nos campos 43 e 44 da Declaração de Óbito indicavam negativa para o óbito ocorrido no ciclo gravídico-puerperal e na causa da morte não constava uma causa materna( ${ }^{(6)}$.

As Declarações de Óbito que não permitiram a identificação do óbito materno ou não materno foram consideradas mortes inconclusivas ${ }^{(6)}$. Essas Declarações de Óbito não apresentaram os campos 43 e 44 preenchidos e as causas de óbito não estavam associadas ao estado gravídico-puerperal.

As mortes presumíveis são caracterizadas por situações em que a causa terminal está atestada, mas não consta na Declaração de Óbito a relação com o estado gravídico-puerperal, não sendo possível identificar o óbito materno ${ }^{(6)}$.

\section{MATERIAL E MÉTODO}

Conduzimos uma pesquisa documental, a partir de prontuários hospitalares de mulheres em idade fértil - de 10 a 49 anos, que foram a óbito no Hospital das Clínicas da Faculdade de Medicina de Ribeirão Preto - Universidade de São Paulo no período de janeiro de 2007 a abril de 2009 .

Solicitamos ao Serviço de Arquivo Médico da instituição o registro de todas as mulheres com idade entre 10 e 49 anos que foram a óbito, no período do estudo, para obtenção dos prontuários hospitalares, nos quais constavam as Declarações de Óbito, objeto desta investigação.

Para a coleta de dados elaboramos um instrumento fundamentado em revisão bibliográfica e no modelo de Declaração de Óbito vigente ${ }^{(6)}$.

Realizamos um estudo piloto, utilizando quinze Declarações de Óbito, a fim de verificar a aplicabilidade do instrumento. Após o piloto, foram realizadas duas reformulações chegando ao instrumento final. Este instrumento nos permitiu obter informações sobre as variáveis sociodemográficas e as variáveis relacionadas ao óbito.

Identificamos, nas Declarações de Óbito, (I) mortes maternas declaradas, (II) mortes não maternas, (III) mortes inconclusivas e (IV) mortes maternas presumíveis. As mortes inconclusivas foram analisadas quanto às causas de óbito. As Declarações de Óbito que apresentavam causas de morte que poderiam ocultar o estado gravídico-puerperal da mulher falecida enquadraram-se nas mortes maternas presumíveis ${ }^{(4)}$. Da mesma forma, classificamos como mortes inconclusivas, as Declarações de Óbito que traziam os campos 43 e 44 assinalados como "sim" - indicando o estado gravídico-puerperal da mulher, mas sem uma causa materna correspondente ao capítulo XV da CID-10 ${ }^{(7)}$, nos campos de causa básica do óbito.

Os dados foram compilados em uma planilha eletrônica utilizando-se o software MS Excel. Para análise das causas 
básicas de morte utilizamos a lista de categorias de três caracteres da $10^{\mathrm{a}}$ Revisão da Classificação de Doenças e Problemas Relacionados à Saúde CID - 10.

O projeto de pesquisa foi aprovado pelo Comitê de Ética do Hospital das Clínicas da Faculdade de Medicina de Ribeirão Preto da Universidade de São Paulo, sob o Protocolo $n^{\circ} 11152 / 2008$. Por se tratar de uma pesquisa de revisão de prontuários dispensou-se o uso do Termo de Consentimento Livre e Esclarecido.

\section{RESULTADOS}

Foram analisadas 301 Declarações de Óbito de mulheres que morreram no Hospital das Clínicas da Faculdade de Medicina de Ribeirão Preto da Universidade de São Paulo no período do estudo. No ano de 2007 foram 131 Declarações de Óbito, em 2008 foram 132 e 38 Declarações de Óbito referentes aos quatro primeiros meses de 2009.

Das 301 Declarações de Óbito analisadas, 180 (60\%) estavam com os campos 43 e 44 preenchidos e 121 (40\%) estavam com os campos 43 e 44 em branco e/ou ignorados. O preenchimento entre os campos variou, haja vista que encontramos mais Declarações de Óbito com o campo 43 preenchido em relação ao campo 44 .

Considerando os campos 43, 44 e a causa da morte, foi possível identificar óbitos maternos declarados, óbitos não maternos, inconclusivos e presumíveis (Figura1).

A partir da análise de 180 Declarações de Óbito com os campos 43 e 44 preenchidos, identificamos que 176 (58,5\%) óbitos ocorreram fora do ciclo gravídico-puerperal, configurando os óbitos não maternos. As quatro declarações restantes, foram classificadas como mortes maternas declaradas. Após análise de 121 Declarações de Óbito com incompletitudes, duas foram identificadas, pela causa básica, como mortes maternas. Estas compuseram, com os quatro óbitos declarados, o grupo de seis ( $2 \%$ ) mortes maternas referentes ao período estudado.

As $119(39,5 \%)$ restantes foram classificadas como mortes inconclusivas e após análise das causas de morte, pudemos identificar que 13 (4,3\%) Declarações de Óbito informavam causas que, de acordo com o Ministério da Saúde ${ }^{(4)}$, poderiam ocultar o estado gestacional, sendo classificadas como mortes maternas presumíveis.
Dentre os 176 (58,5\%) óbitos ocorridos fora do ciclo gravídico-puerperal, um óbito ocorreu no período de 43 dias a 1 ano após o parto de acordo com o preenchimento do campo 44, tendo como causa de morte pneumonia (J18.9 - CID-10).

A partir da análise das causas de morte das Declarações de Óbito classificadas como inconclusivas e de acordo com as diretrizes do Ministério da Saúde, identificamos as causas dos $13(4,3 \%)$ óbitos maternos presumíveis, a saber: pneumonia (3 casos), hipertensão intracraniana (3 casos), choque séptico (2 casos), septicemia (1 caso), broncopneumonia (1 caso), epilepsia (1 caso), tromboembolismo pulmonar (1 caso) e acidente vascular cerebral (1 caso).

Para a análise das causas dos seis ( $2 \%$ ) óbitos maternos foi utilizada a lista de categorias de três caracteres da $10^{\text {a }}$ Revisão da Classificação de Doenças e Problemas Relacionados à Saúde CID 10. Todas as causas maternas declaradas são obstétricas diretas, indicando quatro mortes ocorridas por problemas relativos ao parto, uma por complicações na gestação e uma por complicações puerperais.

\section{DISCUSSÃO}

Apesar do aumento da atenção da Organização Mundial da Saúde, Ministério da Saúde, Secretarias Estaduais e Municipais de Saúde sobre a mortalidade materna nas últimas décadas, a avaliação da redução deste indicador não é simples de ser feita. A mensuração da mortalidade materna é complexa devido à subnotificação dos óbitos e à imprecisão dos registros vitais ${ }^{(8)}$. A redução da mortalidade materna depende do efetivo monitoramento dos casos, fato que nem sempre é possível devido à subinformação, que impede o reconhecimento do óbito como materno ${ }^{(9)}$. Faz-se necessário, portanto, investigar todos os óbitos de mulheres em idade fértil, utilizando mais de uma fonte de informação.

O presente estudo analisou uma das fontes utilizadas na investigação de mortes maternas, a Declaração de Óbito. A qualidade e a confiabilidade das Declarações de Óbito, especialmente das causas relacionadas à gravidez, parto e pós-parto é questionada ${ }^{(10)}$ e estudos apontam fragilidades no preenchimento $^{(5)}$. Um estudo em 15 municípios brasileiros identificou subregistro de $8,7 \%$ e subinformação de $130 \%{ }^{(11)}$, obtendo-se um fator de ajuste da razão da mortalidade materna de 2,3. Outro estudo, sobre o preenchimento dos campos 43 e 44 da

Figura 1 - Mortes segundo a classificação em não maternas, maternas declaradas, inconclusivas e presumíveis, HCFMRP-USP, 2007 a abril de 2009.

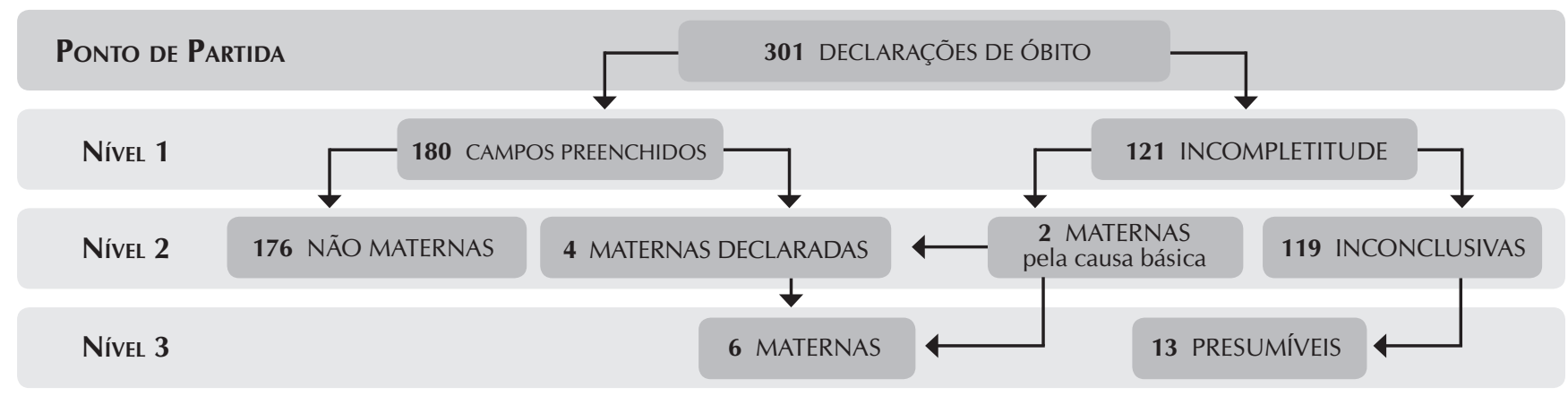


Declaração de Óbito, apontou informação ignorada superior a $87 \%$ para estas variáveis ${ }^{(12)}$. Pesquisa realizada para identificar o preenchimento dos campos 43 e 44, constatou que quase $90 \%$ das Declarações de Óbito estavam com o campo 43 preenchido como ignorado e mais de $90 \%{ }^{(13)}$. No entanto, o Ministério da Saúde declara que o Brasil, de uma maneira geral, conta com uma cobertura adequada do Sistema de Informações sobre Mortalidade e que a subenumeração de óbitos não exceda a $20 \%^{(14)}$.

Outro aspecto que merece discussão é que o item da Declaração de Óbito referente ao intervalo de tempo entre o parto e a morte, geralmente não é preenchido, apesar de haver normas e espaços na Declaração de Óbito tais informações ${ }^{(15)}$. Como consequência, os técnicos da vigilância epidemiológica não podem classificar os óbitos adequadamente, pois casos declarados como mortes maternas se confundem com aqueles ocorridos após um ano do parto ${ }^{(16)}$.

Embora tenhamos chegado ao número de seis óbitos maternos, em decorrência do preenchimento correto dos campos 43, 44 e da causa básica da morte, gostaríamos de enfatizar o que pesquisadores renomados já divulgaram sobre o preenchimento destes campos $^{(17)}$. O preenchimento dos campos 43 e 44, sem que haja menção a uma causa básica materna nas partes I e/ou II da Declaração de Óbito, não significa óbito materno. O correto preenchimento é fundamental e apresenta fortes indícios de morte materna ${ }^{(5)}$.

Quanto aos óbitos declarados como maternos, estudo realizado no Nordeste do país mostra que $50 \%$ das mortes maternas foram diretas. As principais causas foram os transtornos hipertensivos, infecção puerperal e complicações puerperais ${ }^{(18)}$. No mundo todo, as principais causas de mortes maternas incluem hemorragia, eclampsia, sepse, obstrução no trabalho de parto e complicações secundárias a abortos. Estudo realizado em Mali mostra que as principais causas de mortes maternas são as obstétricas diretas, como rotura uterina $(25.7 \%)$, hemorragia no pós-parto $(21.0 \%)$ e infecção pós-parto $(19.8 \%)^{(19)}$.

Quanto à natureza dos demais óbitos, aqueles classificados como inconclusivos foram analisados, sendo identificadas treze mortes presumíveis. Entre as causas, encontramos pneumonia, hipertensão intracraniana, choque séptico, epilepsia, tromboembolismo pulmonar e acidente vascular cerebral. Estudo realizado com dados do Sistema de Internação
Hospitalar do SUS encontrou, dentre as mortes maternas presumíveis de cinco estados do Brasil, um predomínio de doenças cardiovasculares, seguida por complicação pulmonar, doenças cerebrovasculares, hemorragia, edema pulmonar, septicemia e choque hipovolêmico ${ }^{(20)}$. Estudo desenvolvido no Paraná encontrou, entre as causas básicas dos óbitos maternos não declarados, aquelas do grupo de doenças do aparelho circulatório e do aparelho respiratório que no conjunto ocultavam $40 \%$ das mortes maternas.

A Organização Mundial da Saúde declara que, no Brasil, há uma deficiência de registro completo e adequado, mas com outros tipos de dados disponíveis, ou seja, uma posição intermediária em termos de qualidade dos dados ${ }^{(12)}$. Especialistas mostram que medidas foram tomadas para que as Declarações de Óbito sejam mais bem utilizadas. Como exemplo, há o estímulo e o aprimoramento do preenchimento da Declaração de Óbito, com ênfase no preenchimento da declaração da causa da morte, a gratuidade dos registros civis, a introdução de mais uma via na Declaração de Óbito(17). Vários municípios tem conseguido corrigir os dados de mortes maternas e divulgá-los por meio do Sistema de Informação de Mortalidade $^{(16)}$.

\section{CONCLUSÕES}

Esta investigação nos mostrou que o preenchimento de Declarações de Óbito de mulheres em idade fértil, na instituição do estudo, necessita de ajustes para que um diagnóstico mais fidedigno da mortalidade materna seja estabelecido. O subregistro limita o conhecimento da magnitude da ocorrência de mortes maternas, comprometendo o desenvolvimento, implementação e avaliação de ações para reduzir a ocorrência das mortes.

Não podemos nos furtar de reiterar a importância do médico neste cenário, uma vez que é o responsável legal pelo preenchimento da Declaração de Óbito. Os órgãos formadores e associações de classe podem contribuir na capacitação do médico para o preenchimento adequado e completo da Declaração de Óbito. O preenchimento dos campos 43 e 44 da Declaração de Óbito e a confirmação do óbito como materno é de fundamental importância para as estatísticas vitais, para os Sistemas de Informação em Saúde e no final desta cadeia de eventos, para a comunidade.

\section{REFERÊNCIAS}

1. Tajik P, Nedjat S, Afshar NE, Changizi N, Yazdizadeh B, Azemikhah $\mathrm{A}$, et al. Inequality in maternal mortality in Iran: an ecologic study. Int J Prev Med 2012;3(2):116-21.

2. Berg CJ. From identification and review to action-maternal mortality review in the United States. Semin Perinatol 2012;36(1):7-13.

3. Barbuscia DM, Rodrigues-Júnior AL. Completude da informação nas declarações de nascido vivo e nas declarações de óbito, neonatal precoce e fetal, da região de Ribeirão Preto, São Paulo, Brasil, 2000-2007. Cad Saúde Pública 2011;27:1192-200.

4. Ministério da Saúde. Guia de Vigilância Epidemiológica do Óbito Materno. Brasília, DF: O Ministério; 2009.

5. Soares VMN, Azevedo EMM, Watanabe TL. Subnotificação da mortalidade materna no Estado do Paraná, Brasil: 1991-2005. Cad Saúde Pública 2008;24(10):2418-26. 
6. Ministério da Saúde. Manual dos comitês de mortalidade materna. Brasília, DF: O Ministério; 2007.

7. Laurenti R, Mello-Jorge MHP, Gotlieb SLD. Reflexões sobre a mensuração da mortalidade materna. Cad Saúde Pública 2000;16(1):23-30.

8. Mota SMM, Gama SGN, Theme Filha MM. A investigação do óbito de mulher em idade fértil para estimar a mortalidade materna no Município de Belém, Estado do Pará, Brasil. Epidemiol Serv Saúde 2009;18(1):55-64.

9. Leite RMB, Araújo TVB, Albuquerque RM, Andrade ARS, Duarte Neto PJ. Fatores de risco para mortalidade materna em área urbana do Nordeste do Brasil. Cad Saúde Pública 2011;27(10):1977-85.

10. Organização Mundial de Saúde. Beyond the numbers: reviewing maternal deaths and complications to make pregnancy safer. Geneva: WHO; 2004.

11. Haddad SM, Cecatti JG, Parpinelli MA, Souza JP, Costa $M L$, Sousa $M H$, et al. From planning to practice: building the national network for the surveillance of severe maternal morbidity. BMC Public Health 2011;11:283.

12. Morse ML, Fonseca SC, Barbosa MD, Calil MB, Eyer FPC. Mortalidade materna no Brasil: o que mostra a produção científica nos últimos 30 anos? Cad Saúde Pública 2011;27(4):623-38.

13. Laurenti R, Jorge MHP, Gotlieb SLD. Mortes Maternas no
Brasil: Análise do Preenchimento de Variável da Declaração de Óbito. Inf Epidemiol SUS 2000;9(1):43-50.

14. Ministério da Saúde. Estudo da mortalidade de mulheres de 10 a 49 anos, com ênfase na mortalidade materna: relatório final. Brasília, DF: O Ministério; 2006.

15. Laurenti R, Jorge MHPM, Gotlieb SLD. Mortes maternas e mortes por causas maternas. Epidemiol Serv Saúde 2008; 17(4):283-92.

16. Luizaga CTM, Gotlieb SLD, Jorge MHPM, Laurenti $R$. Mortes maternas: revisão do fator de correção para os dados oficiais. Epidemiol Serv Saúde 2010;19(1):8-15.

17. Laurenti R, Jorge MHPM, Gotlieb SLD. A mortalidade materna nas capitais brasileiras: algumas características e estimativa de um fator de ajuste. Rev Bras Epidemiol 2004;7(4):449-60.

18. Correia RA, Araújo HC, Furtado BMA, Bonfim C. Características epidemiológicas dos óbitos maternos ocorridos em Recife, PE, Brasil (2000-2006). Rev Bras Enferm 2011;64:91-7.

19. Teguete I, Maiga AW, Leppert PC. Maternal and neonatal outcomes of grand multiparas over two decades in Mali. Acta Obstet Gynecol Scand 2012;91(5):580-6.

20. Gomes FA, Mamede MV, Costa Júnior ML, Nakano AMS. Morte materna mascarada: um caminho para sua identificação. Acta Paul Enferm 2006;19(4):387-93. 